\title{
Tierversuche - immer mehr wissenschaftliche Studien bezweifeln deren Nutzen
}

\section{Deutsch, B. Lenhard,} F. P. Gruber

Korrespondenz:

Dr. med. Markus Deutsch

Vorstand der Ärztinnen und Ärzte für Tierschutz in der Medizin

Facharzt für Innere Medizin FMH Dürntnerstrasse 20

CH-8340 Hinwil

vorstand@aerztefuertierschutz.ch www.aerztefuertierschutz.ch

\section{Einleitung}

In den letzten Jahren sind in renommierten Fachzeitschriften zahlreiche wissenschaftliche Studien erschienen, die das bisher von der Ärzteschaft weitgehend akzeptierte Konzept des unverzichtbaren Tierversuchs massiv in Frage stellen.

An dieser Stelle möchten wir deshalb einige der erstaunlichen Aussagen dieser Artikel wiedergeben. Wir verzichten bewusst auf eine eigene Wertung. In wenigen Sätzen fassen wir die Arbeiten in chronologischer Reihenfolge zusammen und lassen die Originaltexte für sich sprechen.

\section{British Medical Journal 2007}

Perel P, Roberts I, Sena E, Wheble P, Briscoe C, Sandercock $P$, et al. Comparison of treatment effects between animal experiments and clinical trials: systematic review. BMJ. 2007;334(7586):197. Epub 2006 Dec 15

Diese Literaturübersicht aus dem Jahr 2007 (Publikation im Internet bereits am 15. Dezember 2006, Vorabdrucke noch unter dem Titel «Testing treatments on animals: relevance to humans») untersuchte die Übereinstimmung der Ergebnisse klinischer Studien und der entsprechenden Tierversuche.

Hierzu identifizierten die Autoren sechs Interventionen mit gesichertem klinischem Effekt und verglichen diesen mit dem Effekt in Tierversuchen. Ausdrücklich betont wird, dass die Auswahl dieser Interventionen erfolgte, bevor das Resultat der Tierversuche den Untersuchern bekannt war.

Die sechs untersuchten Gebiete waren Schädel-Hirn-Trauma, Antifibrinolytica bei Hämorrhagie, Thrombolyse bei akutem ischämischem Hirnschlag, Tirilazad bei akutem ischämischem Hirnschlag, pränatale Steroidgabe zur Verhütung des neonatalen Respiratory Distress Syndrome und Bisphosphonate zur Therapie der Osteoporose.

Die Studie fand bei drei Interventionen ähnliche Ergebnisse, bei den drei übrigen hingegen nicht.

Die Arbeit gelangte zum Schluss, dass

- die methodische Qualität der Tierversuchsstudien insgesamt schlecht war;

\section{Les études scientifiques contes-} tant l'utilité de l'expérimentation animale sont de plus en plus

\section{nombreuses}

Ces dernières années, des revues spécialisées renommées ont publié de nombreuses études scientifiques mettant en question le concept de l'inévitable expérimentation animale, largement accepté jusqu'à présent par le corps médical. C'est pourquoi, nous aimerions reproduire dans les lignes qui suivent quelques constatations étonnantes faites par ces études. Nous renonçons sciemment à émettre un jugement de valeur. Nous résumons en quelques phrases les travaux de manière chronologique et laissons les textes originaux parler d'euxmêmes.

Les études présentées parviennent aux conclusions suivantes:

1. L'expérimentation animale ne correspond souvent pas aux exigences de travaux menés proprement sur le plan scientifique.

2. L'expérimentation animale contredit (trop) souvent les résultats d'études cliniques.

3. L'expérimentation animale est souvent effectuée simultanément avec des études cliniques ou même selon de telles études, ce qui sape la croyance en la nécessité d'une telle expérimentation avant toute application sur l'être humain.

4. Les travaux scientifiques prouvent qu'aujourd'hui encore, de nombreuses expérimentations animales sont effectuées inutilement.

5. Pour améliorer la qualité de l'expérimentation animale, notamment pour empêcher la «publication bias», l'introduction de registres d'études doit être exigée aussi pour l'expérimentation animale. 
- starke Hinweise für «publication bias» vorlagen («The animal studies were of poor quality, however, with evidence of publication bias.»);

- dieser «publication bias» durch Studienregister analog denjenigen in der klinischen Medizin hätte vermieden werden können: «Prospective registration of animal experiments might prevent publication bias»;

- die Ergebnisse der Tierversuche insgesamt zu oft den klinischen Ergebnissen widersprachen;

- die fehlende Übereinstimmung der Tierversuche mit den klinischen Versuchen die Bedeutung der Tierversuche in Frage stellt;

- diese fehlende Übereinstimmung bedingt sein könnte durch «bias» oder durch ungenügende Wiedergabe der klinischen Situation durch den Tierversuch: «Lack of concordance between animal experiments and clinical trials may be due to bias, random error, or the failure of animal models to adequately represent human disease».

Weiter bemängelte die Arbeit die Zusammenarbeit zwischen klinischen und tierexperimentellen Forschern: «Several of our cases alluded to a lack of communication between those conducting animal experiments and those conducting the corresponding clinical trials.»

Diese Arbeit wurde auch im Periskop der Zeitschrift

\section{Schweizerisches Medizin-Forum 2007}

Schweiz Med Forum. 2007;7:530.

aufgenommen und besprochen mit dem Fazit: «Die Diskrepanzen zeigen, dass der Tierversuch nichts oder nur wenig mit der menschlichen Krankheit zu tun hat! Und oftmals sind Tierversuche von zweifelhafter Qualität. Probleme und Zweifel am Tierversuch!»

\section{British Medical Journal 2004}

Pound P, Ebrahim S, Sandercock P, Bracken MB, Roberts I. Reviewing Animal Trials Systematically (RATS) Group. Where is the evidence that animal research benefits humans? BMJ. 2004;328:514-7.

Auch diese Arbeit aus England ging der Frage nach, ob es Evidenz gibt für den Nutzen von Tierversuchen in der Medizin. Die Forscher suchten systematische Reviews zum Nutzen von Tierversuchen und fanden sechs Reviews, die explizit der Frage nachgingen, wie die Tierversuche die klinischen Versuche beeinflusst hatten.

Eines der erstaunlichsten Ergebnisse war folgendes: In mehreren Studien fanden sie, dass Studien gleichzeitig beim Menschen wie am Tier durchgeführt wurden! («The few existing reviews have highlighted deficiencies such as animal and clinical trials being conducted simultaneously.»)

Dies führte zu einem folgenschweren Schluss:

«This suggests that the animal data were regarded as irrelevant, calling into question why the studies were done in the first place and seriously undermining the principle that animal experiments are necessary to inform clinical medicine.»

Die Methodik der Studien beurteilten sie wie folgt: «Furthermore, many of the existing animal experiments were poorly designed.»

Und weiter: «Many animal studies were of poor methodological quality.»

Sie forderten deshalb: «Systematic reviews should become routine to ensure the best use of existing animal data as well as improve the estimates of effect from animal experiments.»

Das Fazit dieser Arbeit zu Tierversuchen ganz allgemein fiel noch viel grundsätzlicher aus:

«Ideally, new animal studies should not be conducted until the best use has been made of existing animal studies and until their validity and generalisability to clinical medicine has been assessed.»

In dieser Arbeit wird auf verschiedene frühere Arbeiten eingegangen, die sich mit dem Nutzen von Tierversuchen beschäftigen. Unter anderen wird auch auf die weiter unten beschriebenen Arbeiten aus Stroke 2001 und Stroke 1990 eingegangen.

\section{ALTEX 2006}

Lindl T. Study of the clinical relevance of 51 applications on animal experiments in biomedical research. ALTEX. 2006;23(2):112.

und

\section{ALTEX 2001}

Lindl T, Weichenmeier I, Labahn D, Gruber FP, Völkel M. Evaluation von genehmigten tierexperimentellen Versuchsvorhaben in Bezug auf das Forschungsziel, den wissenschaftlichen Nutzen und die medizinische Relevanz. ALTEX. 2001;18(3):171-8. Diese interessante Studie aus dem Jahr 2001 mit einem Follow-up 2006 ging der Frage nach, ob der bei der Beantragung eines Tierversuchs angegebene Nutzen je erreicht wird.

Dies ist einer der Hauptkritikpunkte aller Tierversuchsskeptiker. Im Rahmen des Genehmigungsverfahrens muss der Forscher glaubhaft darlegen, welchen Nutzen sein Tierversuch im Vergleich zum sicheren Leiden/Tod für das Tier bringen wird. Der Nutzen kann relativ leicht postuliert werden, und der Beweis des fehlenden Nutzens ist äusserst schwer zu erbringen, da er meist erst nach Jahren bis Jahrzehnten beurteilt werden kann. Bis dann interessiert sich jedoch 
niemand und auch keine Behörde mehr für einen mittlerweile dermassen verjährten Versuch.

Untersucht wurden 51 in Bayern genehmigte Tierversuchsanträge zwischen 1991 und 1993. Evaluiert wurde einerseits, ob die Belastung für die Tiere im Versuch wirklich den Angaben im Versuchsantrag entsprach. Es zeigte sich, dass in zwei Dritteln der Versuche die Belastung der Tiere im Antrag zu niedrig eingeschätzt worden war.

Zweitens wurde untersucht, ob die Projekte je zu wissenschaftlich weiterführenden Erkenntnissen beitrugen. Von den 51 bewilligten Tierversuchen waren 35 ausdrücklich vom Antragsteller nicht als Grundlagenforschung deklariert. Trotzdem erreichten von diesen 35 nur 8 das angegebene Versuchsziel, und 3 erreichten es teilweise. Erstaunlich war weiter, dass das Versuchsziel um so häufiger erreicht wurde, je geringer die Belastung der Tiere im Versuch war und je evolutiv niedriger die gewählte Versuchstierart war. Insbesondere erreichte kein einziger der Versuche mit schwerster Belastung für das Tier das Versuchsziel.

Weiter ergab sich ein Zusammenhang zwischen der Qualität des Versuchsantrags und dem Erreichen des Versuchsziels. Die Autoren kamen zum Schluss, dass Anträge mit Standardformulierungen nur mit der Häufigkeit von Zufallstreffern für den Menschen relevante Erkenntnisse lieferten.

Das Fazit dieser Forscher: «Wir kommen zu der Annahme, dass offensichtlich Erkenntnisse aus Tierversuchen nicht das halten, was (sich) Tierexperimentatoren für den Menschen versprechen.»

Sie fordern deshalb:

- «Versuchsvorhaben, die dem Tier schwere Leiden (Belastungsgrad 2) verursachen, sind besonders $\mathrm{zu}$ begründen. Der Belastungsgrad 3 (schwerste Belastung) sollte in keinem Fall mehr genehmigt werden.»

- «Jedes genehmigte Versuchsvorhaben ist einer strikten Erfolgskontrolle zu unterwerfen. Diese ist den Mitgliedern der Tierversuchskommission zugänglich zu machen.»

Die Nachfolgearbeit 2006 ging der Frage nach, ob die Resultate der Tierversuche mittlerweile Eingang in die Medizin gefunden hatten. Untersucht wurde dies mittels Zitierhäufigkeit, Zitierverlauf sowie der Kontrolle, ob die Arbeiten in klinische Studien oder Reviews Eingang gefunden hatten.

Das Ergebnis war erschütternd: Nur bei 0,3\% der Studien wurde ein Zusammenhang zwischen tierexperimentellen Befunden der Antragsteller und den Ergebnissen beim Menschen herge- stellt. Schlimmer noch: Selbst bei diesen wenigen Studien konnte die im Tierversuch bestätigte Hypothese nicht in eine neue Therapie beim Menschen umgesetzt werden, da entweder kein therapeutischer Effekt nachweisbar war oder die Befunde am Menschen denjenigen des Tierversuchs widersprachen.

«[...] nur bei 4 Studien $(0,3 \%)$ wurde ein direkter $\mathrm{Zu-}$ sammenhang zwischen tierexperimentellen Befunden der Antragsteller und den gefundenen Ergebnissen am Menschen hergestellt.

Doch selbst hier konnte die im Tierversuch bestätigte Hypothese klinisch nicht in eine neue Therapie am Menschen umgesetzt werden.

Entweder war kein therapeutischer Effekt nachweisbar oder die Befunde am Menschen widersprachen sogar den Ergebnissen am Tier.»

\section{Acad Emerg Med 2003}

Bebarta V, Luyten D, Heard K. Emergency medicine animal research: does use of randomization and blinding affect the results? Acad Emerg Med. 2003; 10(6):684-7.

In dieser Arbeit gingen die Forscher der Frage nach, ob Tierversuche den Anforderungen an eine wissenschaftlich saubere Methodik genügen. Bei klinischen Studien ist es heute Standard, diese doppelblind und randomisiert durchzuführen. Nicht hingegen bei Tierversuchen. Fehlendes Blinding und fehlende Randomisierung führen nachgewiesenermassen zu einer Überschätzung eines Effekts gegenüber klinischen Studien mit diesen Massnahmen. Die Autoren untersuchten deshalb, ob diese zwei Studientechniken nicht doch auch einen Einfluss auf Tierversuche haben.

Sie fanden: «These results suggest that animal studies that do not utilize Randomization and Blinding are more likely to report a difference between study groups than studies that employ these methods.»

\section{British Medical Journal 2002}

Roberts I, Kwan I, Evans P, Haig S. Does animal experimentation inform human healthcare? Observations from a systematic review of international animal experiments on fluid resuscitation. BMJ. 2002;324:474-6.

Auch diese Arbeit befasste sich mit der methodischen Sauberkeit von Tierversuchen anhand eines Reviews der Literatur zum Thema «fluid resuscitation in uncontrolled haemorrhage». Sie fanden wesentliche methodologische Mängel und forderten eine bessere Dokumentation, Randomisierung, statistische Methodologie und insbesondere ein vorgängiges systematisches Review der entsprechenden Literatur. 
«A systematic review of animal experiments on fluid resuscitation found that most studies were underpowered and provided little information on possible bias.»

«Systematic reviews of animal experiments would help to ensure that animal experiments do not set out to answer questions that have already been answered, reduce bias and increase precision, and provide reassurance about whether the results can be generalised.»

«Prospective registration of animal experiments would help to avoid publication bias.»

«On methodological grounds, animal experimentation would better contribute to human health care if we promoted registration, randomisation, and systematic reviews.»

\section{Stroke 2001}

Horn $J$, de Haan RJ, Vermeulen $M$, Luiten $P G$, Limburg M. Nimodipine in animal model experiments of focal cerebral ischemia: a systematic review. Stroke. 2001;32:2433-8.

Diese Arbeit ging der Frage nach, warum sich Nimodipin in klinischen Studien als nicht wirksam erwiesen hatte, obwohl vorgängige Tierversuche vielversprechend gewesen waren.

Sie kam zum Schluss, dass die Tierversuche von schlechter methodischer Qualität waren und der effektiv geringe Nutzen von Nimodipin so klein war, dass klinische Studien in grossem Rahmen gar nie hätten angeschlossen werden dürfen.

Überraschenderweise fanden sie auch, dass klinische Versuche teils gleichzeitig mit den Tierversuchen liefen statt danach, wie man es erwarten würde, wenn die vorgängigen Tierversuche wichtig wären für die klinische Anwendung.

«We conclude that the results of the animal experiments reviewed in the present investigation did not show convincing empirical evidence to substantiate the decision for trials with nimodipine in stroke patients.»

«Surprisingly, we found that animal experiments and clinical studies ran simultaneously.»

Hieraus kann man nur schliessen, dass die Forscher selbst nicht an die Relevanz der Tierver- suche glaubten und deshalb beschlossen, Nimodipin so oder so am Menschen zu erproben. Weiter heisst dies aber auch, dass die Tierversuche keinerlei Konsequenzen hatten und somit nicht gerechtfertigt waren.

Als Schlusswort wissenschaftlicher Publikationen zur Relevanz von Tierversuchen wollen wir ein Editorial in Stroke zitieren:

\section{Stroke 1990}

Wiebers DO, Adams HP Jr, Whisnant JP. Animal models of stroke: are they relevant to human disease? Stroke. 1990;21(1):1-3.

«Ultimately, however, the answers to many of our questions regarding the underlying pathophysiology and treatment of stroke do not lie with continued attempts to model the human situation perfectly in animals, but rather with the development of techniques to enable the study of more basic metabolism, pathophysiology, and anatomical imaging detail in living humans.»

\section{Zusammenfassung}

Die hier aufgeführten Studien kommen zu diesen Schlussfolgerungen:

1. Tierversuche entsprechen oft nicht den Anforderungen an wissenschaftlich sauberes Arbeiten.

2. Tierversuche widersprechen (zu) oft den Ergebnissen klinischer Studien.

3. Tierversuche werden oft gleichzeitig mit oder gar nach klinischen Studien durchgeführt. Dies untergräbt den Glauben an die Notwendigkeit der Tierversuche vor der Anwendung am Menschen.

4. Diese wissenschaftlichen Arbeiten beweisen, dass auch heute noch zahlreiche unnötige Tierversuche durchgeführt werden.

5. Zur Verbesserung der Qualität der Tierversuche, insbesondere zur Vermeidung des «publication bias», ist die Einführung von Studienregistern auch für Tierversuche zu fordern. 